\title{
Phase II clinical study of modified FOLFOX7 (intermittent oxaliplatin administration) plus bevacizumab in patients with unresectable metastatic colorectal cancer-CRAFT study
}

\author{
Tohru Tezuka • Chikuma Hamada • Hideyuki Ishida • Mitsuru Ooshiro • \\ Hiroshi Matsuoka - Shingo Kawasaki - Hideyuki Mishima $\cdot$ Kotaro Maeda • \\ Junichi Sakamoto $\cdot$ Keiji Koda
}

Received: 9 April 2013 /Accepted: 13 May 2013 / Published online: 2 July 2013

(C) The Author(s) 2013. This article is published with open access at Springerlink.com

\begin{abstract}
Summary Purpose Continuous treatment with FOLFOX therapy is associated with peripheral nerve toxicity, and to improve this inconvenient side effect various methods of administration are being investigated. A regimen of intermittent oxaliplatin administration by continuous infusion therapy, i.e., modified FOLFOX7 (mFOLFOX7) + bevacizumab, was designed with the goal of alleviating severe peripheral nerve disorders and hematological toxicity. A phase II clinical study
\end{abstract}

T. Tezuka $(\bowtie) \cdot$ K. Koda

Department of Surgery, Teikyo University Chiba Medical Center, 3426-3 Anesaki,

Ichihara City, Chiba, Japan 299-0111

e-mail: tohru_tezuka@tokyo-hmt.jp

C. Hamada

Faculty of Engineering, Tokyo University of Science, Tokyo, Japan

\section{H. Ishida}

Department of Digestive Tract and General Surgery, Saitama

Medical Center, Saitama Medical University, Saitama, Japan

\section{Ooshiro}

Department of Surgery, Toho University Sakura Medical Center,

Chiba, Japan

H. Matsuoka $\cdot$ K. Maeda

Department of Surgery, Fujita Health University Hospital, Aichi, Japan

S. Kawasaki

Department of Surgery, Saishukan Hospital, Aichi, Japan

H. Mishima

Aichi Medical University Cancer Center, Aichi, Japan

J. Sakamoto

Tokai Central Hospital, Gifu, Japan was conducted to evaluate the efficacy and safety of this regimen. Methods Previously untreated patients were assigned to $\mathrm{mFOLFOX7}$ (oxaliplatin $85 \mathrm{mg} / \mathrm{m}^{2}$, levofolinate [l-LV] $200 \mathrm{mg} / \mathrm{m}^{2}$, 5-fluorouracil [5-FU] $2400 \quad \mathrm{mg} / \mathrm{m}^{2}$ ) + bevacizumab $(5 \mathrm{mg} / \mathrm{kg})$ administered every 2 weeks for 8 cycles, maintenance without oxaliplatin for 8 cycles, and reintroduction of mFOLFOX7 + bevacizumab for 8 cycles or until disease progression. Progression free survival (PFS) following the first dose (PFS 1) and following reintroduction of oxaliplatin (PFS 2) were used as indices for assessing the efficacy of intermittent administration. Results Fifty-two patients were enrolled, with median age of 64 years (range, 3674). Median PFS 1 was 11.8 months (95\% confidence interval [CI], 9.5 to 13.7), median time to treatment failure was 10.3 months ( $95 \%$ CI, 5.6 to 12.1), percentage of patients with neutropenia of grade 3 or higher was $7.8 \%$, and percentage with peripheral nerve disorders was $3.9 \%$. Response rate was $50 \%$, and $84.4 \%$ of patients who started modified simplified LV5FU2 + bevacizumab were reintroduced to oxaliplatin. Conclusion By excluding 5-FU bolus administration and administering bevacizumab continuously the mFOLFOX7 + bevacizumab regimen with preplanned withdrawal of oxaliplatin showed high tolerability and prevented severe peripheral neuropathy and neutropenia without reducing efficacy.

Keywords Oxaliplatin · Bevacizumab · Stop-and-go · Modified FOLFOX7 $\cdot$ Metastatic colorectal cancer

\section{Introduction}

In the 1980s, 5-fluorouracil (5-FU)/leucovorin (LV) was the standard recommended first-line therapy for unresectable 
metastatic colorectal cancer (mCRC) $[1,2]$. Since then, irinotecan and oxaliplatin have been developed, and 5FU/LV/oxaliplatin (FOLFOX) [3-5] and 5-FU/LV/irinotecan (FOLFIRI) [6] are now recommended in the NCCN Guidelines in addition to 5-FU/LV therapy. Hurwitz et al. reported prolonged survival by combining bevacizumab with irinotecan plus bolus 5-FU (IFL) [7], and combinations of chemotherapy with several molecular targeting agents are now currently recommended in the NCCN Guidelines and in the guidelines from other countries. Prolonged survival as an additive effect of molecular targeting agents has been reported in first-line therapy with 5-FU/LV plus bevacizumab [8], and the additive effect of bevacizumab to an oxaliplatin-based regimen was demonstrated in the NO16966 study [9].

As described above, 5-FU/LV plus bevacizumab and FOLFOX plus bevacizumab have become established as standard, first-line therapies for unresectable mCRC. However, peripheral neuropathy and serious hematological toxicity, which are cumulative toxicities of oxaliplatin in FOLFOX, have been obstacles to continuous therapy [10]. To address this issue, oxaliplatin withdrawal (the stop-andgo concept) was systematically incorporated into the OPTIMOX1 study [11] and the OPTIMOX2 study [12] and was shown to be useful. Subsequently, a randomized phase III clinical study of bevacizumab combined with FOLFOX (the CONcePT trial) was conducted in order to validate the stop-and-go concept and to examine the incidence of peripheral neuropathy depending on the administration of calcium gluconate and magnesium sulfate $(\mathrm{Ca} / \mathrm{Mg}$ formulation), with results reported at the American Society of Clinical Oncology Annual Meeting in 2008 [13]. In that study, continuous administration of oxaliplatin (continuous oxaliplatin; $\mathrm{CO}$ ) and intermittent administration of oxaliplatin (intermittent oxaliplatin; IO) were compared using modified FOLFOX7 (mFOLFOX7; oxaliplatin $85 \mathrm{mg} / \mathrm{m}^{2}$ ) plus bevacizumab. Median time to treatment failure (TTF), the primary endpoint, was 4.2 months in the $\mathrm{CO}$ arm and 5.6 months in the IO arm (HR, 0.58; $P=0.0025$ ), demonstrating that the IO arm was significantly better. The incidence of peripheral neuropathy of grade 3 or higher, the rate of discontinuation of therapy due to peripheral neuropathy, and the need to delay the treatment and/or reduce dose were all lower in the IO arm. That report suggested the oxaliplatin stop-and-go concept to be favorable even when bevacizumab was combined with FOLFOX, but it has not been established as the standard of care, and the clinical question of reintroducing oxaliplatin remains.

Therefore, we conducted the phase II CRAFT (Confirmation of Avastin and intermittent mFOLFOX7 Therapy in advanced colorectal cancer) study in order to examine the efficacy and safety of a regimen of mFOLFOX7 plus bevacizumab with intermittent oxaliplatin administration for Japanese colorectal cancer patients.

\section{Materials and methods}

This study was a multicenter phase II clinical study to examine the efficacy and safety of intermittent oxaliplatin administration in mFOLFOX7 plus bevacizumab as the first-line therapy for patients with unresectable colorectal cancer. The primary endpoint was progression-free survival 1 (PFS 1, the period from the start of the treatment to the point of first progression); secondary endpoints were time to treatment failure (TTF), response rate 1 (RR 1, the response rate during the first oxaliplatin treatment), progression-free survival 2 (PFS 2, the period from the first progression during maintenance to the second progression after reintroduction of oxaliplatin), response rate 2 (RR 2, the response rate during reintroduction of oxaliplatin following the first progression), and the safety.

\section{Patient eligibility}

Patients were entered into the study if they fulfilled the following inclusion criteria: 1) colorectal cancer was definitely diagnosed histologically; 2) the colorectal cancer was unresectable and metastatic; 3) the disease had not been treated with chemotherapy or radiation therapy; 4) at least one lesion was evaluable; 5) age 20 to 79 years; 6) the Eastern Cooperative Oncology Group (ECOG) Performance Status (PS) was 0 or 1; and 7) they had sufficient bone-marrow, liver, and kidney functions. Patients were excluded if there was 1) suspicion of brain metastasis; 2) suspicion of arterial thromboembolism; 3) chronic inflammatory disease such as rheumatoid arthritis; 4) bleeding tendencies; 5) uncontrollable peptic ulcer, hypertension, diarrhea, or infection; 6) heart disease with symptoms; or 7) pregnancy or lactation.

This study was conducted in accordance with the World Medical Association Declaration of Helsinki and the Ethical Guidelines for Clinical Studies. The scientific and ethical validity of this study was reviewed by the Ethical Review Board of each participating facility, and the study was conducted after receiving their approval. Informed consent was obtained from all enrolled patients in writing before enrollment. This study was registered with UMIN-CTR (number: UMIN000002354).

Treatment plan and evaluation

The protocol treatment was started within 14 days after enrollment of patients who were determined to be eligible. On day 1 , bevacizumab $5 \mathrm{mg} / \mathrm{kg}$ was administered by initial 90-min intravenous infusion (which could be shortened to $60 \mathrm{~min}$ at the second time and to $30 \mathrm{~min}$ from the third time), then oxaliplatin $85 \mathrm{mg} / \mathrm{m}^{2}$ and levofolinate $(l-\mathrm{LV})$ $200 \mathrm{mg} / \mathrm{m}^{2}$ were administered by 120 -min intravenous 
Fig. 1 Chemotherapy regimens

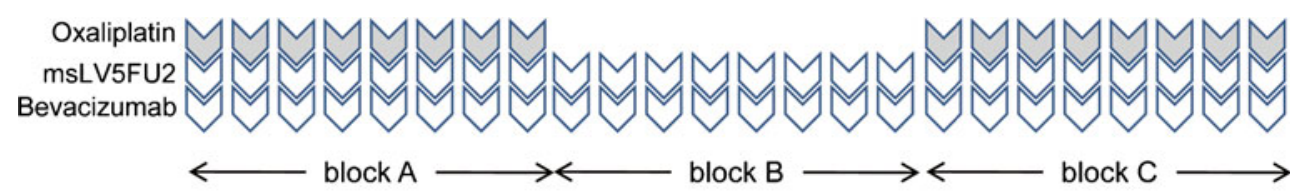

Treatment block $\mathrm{A}$ and $\mathrm{C}$

(mFOLFOX7 + Bevacizumab)

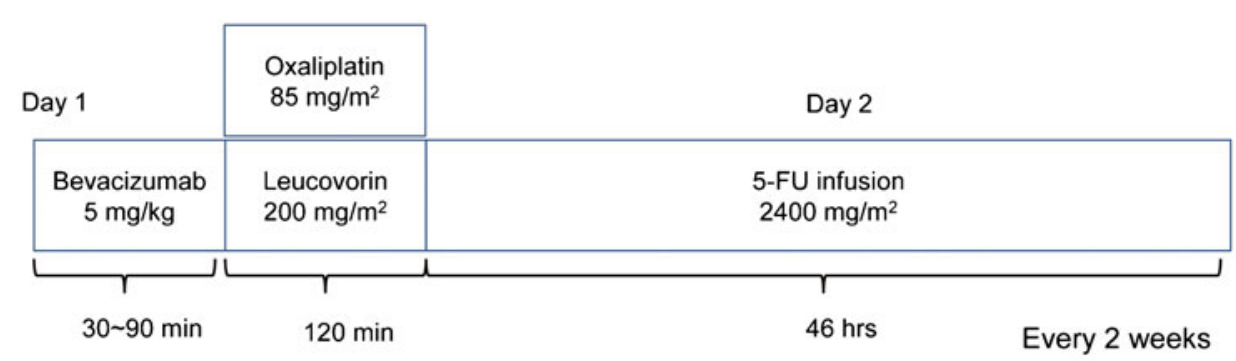

Treatment block B

(msLV5FU2 + Bevacizumab)

Day 1

Day 2

\begin{tabular}{|c|c|c|}
\hline $\begin{array}{l}\text { Bevacizumab } \\
5 \mathrm{mg} / \mathrm{kg}\end{array}$ & $\begin{array}{l}\text { Leucovorin } \\
200 \mathrm{mg} / \mathrm{m}^{2}\end{array}$ & $\begin{array}{l}5-\mathrm{FU} \text { infusion } \\
2400 \mathrm{mg} / \mathrm{m}^{2}\end{array}$ \\
\hline \multicolumn{3}{|c|}{$r$ r } \\
\hline $30 \mathrm{~min}$ & $120 \mathrm{~min}$ & $46 \mathrm{hrs}$ \\
\hline
\end{tabular}

infusion, 5 -FU $2400 \mathrm{mg} / \mathrm{m}^{2}$ was administered by 46-h (days 1-2) continuous intravenous infusion, with 12 days of drug withdrawal thereafter. These 14 days constituted 1 cycle, and cycles 1-8 were conducted (block A). Thereafter, 8 cycles (cycles 9-16) were conducted without oxaliplatin (block B). Then oxaliplatin was reintroduced for 8 cycles (block C), so that a total of 24 cycles was administered as the protocol treatment (Fig. 1). Protocol treatment was continued as long as it did not conflict with the criteria for discontinuing treatment. However, the following cases were defined as the end of protocol treatment:

(1) The 24 cycles (block A followed by block B and then block $\mathrm{C}$ ) prescribed in the protocol had been completed. However, if progressive disease (PD) was confirmed before the completion of 8 cycles in block B,

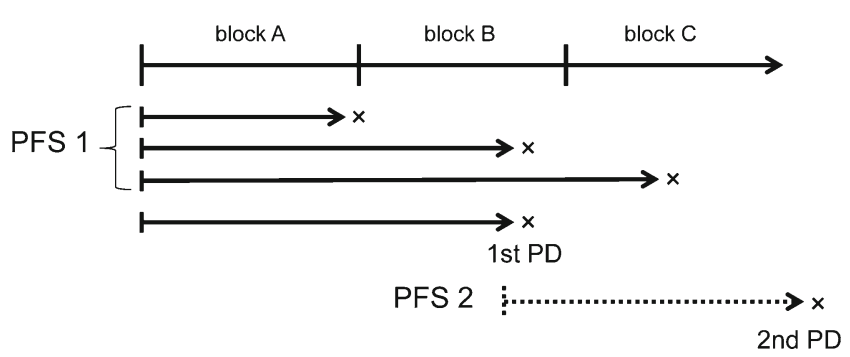

Fig. 2 Definition of progression free survival. PFS 2: first PD designated as starting point the end of protocol was defined as the end of block $\mathrm{C}$ following reintroduction of oxaliplatin.

(2) The patient became operable as a result of the efficacy of treatment (with the day of surgery defined as the end of protocol treatment).

Computerized tomography (CT)/magnetic resonance imaging (MRI) was performed every 8 weeks during the protocol treatment, counting from the day of enrollment, in order to measure and evaluate target lesions, evaluate nontarget lesions, and check for the appearance of new lesions. Image assessment by CT/MRI or other method was also permitted as necessary. If the tumor site was measured by a method other than CT/MRI, the tumor was evaluated before treatment and the same method was continued until PD. As a rule, CT/MRI was also conducted using the same measurement method and the same slice thickness as used in examination at baseline. Evaluation of tumor reduction was according to Response Evaluation Criteria in Solid Tumors (RECIST, version 1.1).

Adverse events were evaluated by the National Cancer Institute Common Terminology Criteria for Adverse Events (NCI-CTCAE v3.0) in all enrolled patients who received the protocol treatment at least once. The frequency and grade of the most serious clinical findings and laboratory test values in each cycle were tabulated. Patients were observed carefully for previously reported typical adverse drug reactions to bevacizumab and oxaliplatin, including hemorrhage, 
Table 1 Baseline patient characteristics

\begin{tabular}{|c|c|}
\hline Characteristics & $n$ \\
\hline Enrolled & 52 \\
\hline \multicolumn{2}{|l|}{ Sex } \\
\hline Male & 32 \\
\hline Female & 20 \\
\hline Age, years median (range) & $64(36-74)$ \\
\hline \multicolumn{2}{|l|}{ ECOG performance status } \\
\hline 0 & 43 \\
\hline 1 & 9 \\
\hline \multicolumn{2}{|l|}{ Primary site } \\
\hline Colon & 25 \\
\hline Rectum & 27 \\
\hline \multicolumn{2}{|l|}{ Histological type } \\
\hline tub1 & \\
\hline tub2 & 36 \\
\hline por & 5 \\
\hline muc & \\
\hline sig & \\
\hline ecc & \\
\hline Miscellaneous carcinoma & 2 \\
\hline \multicolumn{2}{|l|}{ Site of metastasis } \\
\hline Liver & 34 \\
\hline Lung & 21 \\
\hline Lymph node & 12 \\
\hline Peritoneum & 5 \\
\hline \multicolumn{2}{|l|}{ Surgery } \\
\hline Yes & 26 \\
\hline No & 26 \\
\hline
\end{tabular}

ECOG Eastern Cooperative Oncology Group

tubl well differentiated type tubular adenocarcinoma

tub2 moderately differentiated type tubular adenocarcinoma

por poorly differentiated adenocarcinoma

muc mucinous adenocarcinoma

sig signet-ring cell carcinoma

ecc endocrine cell carcinoma

thrombosis, gastrointestinal perforation, increased blood pressure, hemotoxicity, and peripheral neuropathy.

\section{Definition of endpoints}

In this study, the following definitions were used to assess therapeutic effect: PFS 1 (Fig. 2) is the period from the day of enrollment to the confirmation of PD or death from any cause, whichever comes first; TTF is the period from the day of enrollment until the date of discontinuation of treatment, the date of PD confirmation, or the date of death, whichever comes first; RR 1 is the percentage of patients whose best overall response is either CR or PR; PFS 2
(Fig. 2) is the period from the date of first PD during modified simplified LV5FU2 (msLV5FU2) plus bevacizumab to the date of confirmation of PD (second PD after the day of enrollment) after reintroduction of mFOLFOX7 plus bevacizumab, or the date of death from any cause is confirmed, whichever comes first; RR 2 is the response rate during reintroduction of oxaliplatin following the first PD.

Statistical determination of target sample size

In the NO16966 study [9], a phase III clinical study of FOLFOX4 or capecitabine/oxaliplatin (XELOX) with/without bevacizumab, the median PFS by ontreatment analysis excluding PD cases from at least 29 days after the final administration of the study drug was 10.4 months. In the PACCE trial [14], a phase III clinical study conducted to confirm the additive effect of panitumumab to FOLFOX plus bevacizumab, the median PFS for FOLFOX plus bevacizumab was 11.2 months. Therefore, the threshold PFS in this study was assumed to be 7 months considering PFS in the phase III study of FOLFOX4 [15], and the anticipated PFS was set at 10.5 months. Level of significance was 0.05 , power was $80 \%$, and the required number of cases determined by using the Southwest Oncology Group's one-arm survival design (http://www.swogstat.org/statoolsout.html) was 42 patients. The target number of cases in this study was 50 patients, considering a dropout rate of $10 \%$.

Analysis plan

PFS and TTF survival curves were estimated by the KaplanMeier method, median survival time was calculated, and the two-sided $95 \%$ confidence interval was calculated.

As for the response rate, antitumor effect (overall effect) was compiled for patients with measurable lesions among the per protocol set (PPS). Exact confidence interval based on binomial distribution was used for the interval estimation.

\section{Results}

Patient characteristics

Fifty-two patients from 18 institutions were enrolled from February 2009 to June 2010. Four out of the 52 patients were excluded as subjects of the efficacy analysis due to ineligibility after enrollment; therefore, the efficacy analysis was conducted with 48 patients. The safety analysis set included 51 patients, all enrolled patients who received the protocol treatment at least once. Data cutoff was May 2011, and the median observation period was 9.3 months. Baseline patient 
Fig. 3 Study profile

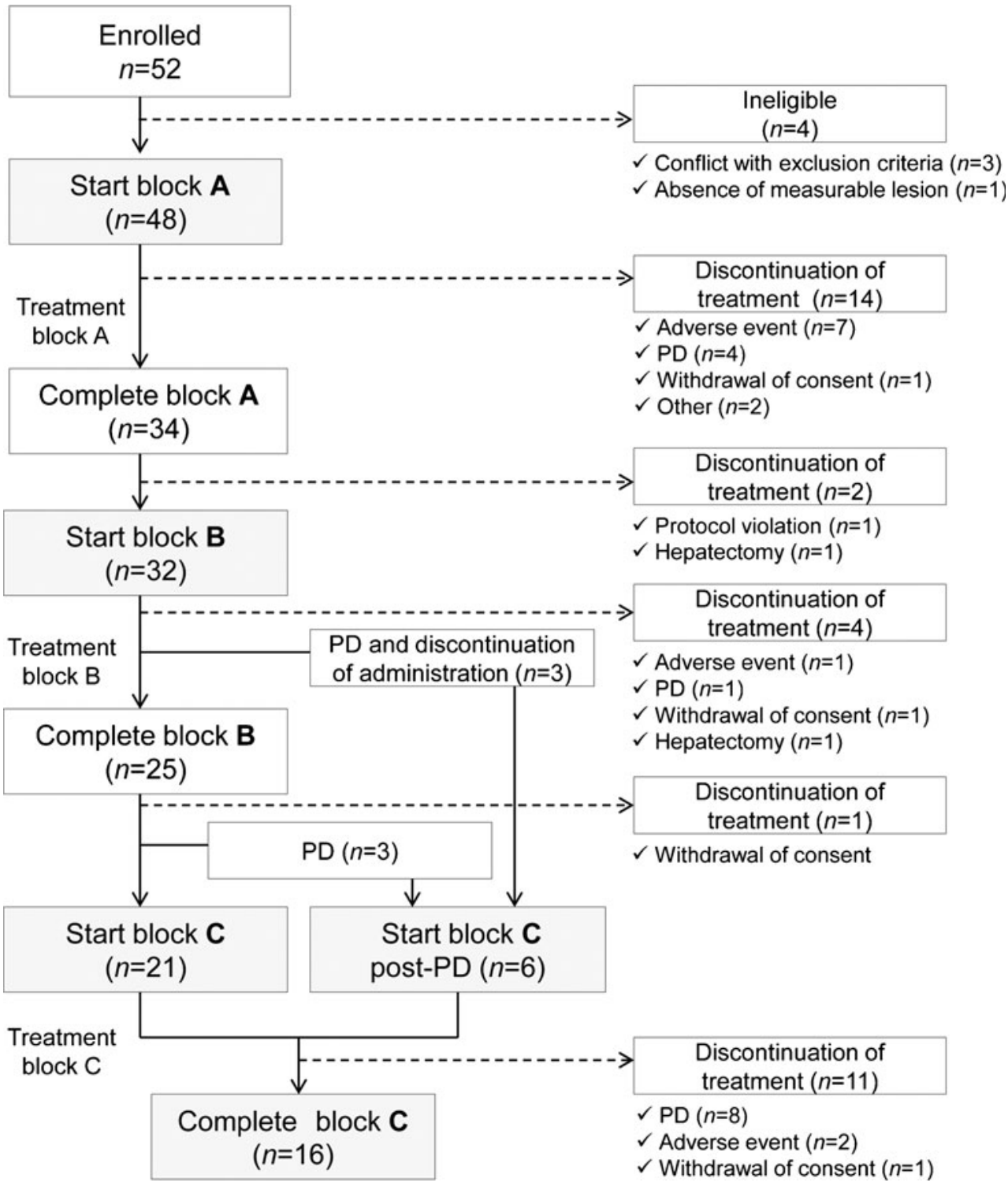

characteristics are shown in Table 1 . Median age was 64 years (36-74), male/female ratio was 32/20, colon cancer/rectal cancer ratio was 25/27, and ECOG PS 0/1 ratio was 43/9.

\section{Treatment status}

The study profile of protocol treatment is shown in Fig. 3 . Of the 52 patients enrolled, 48 started the protocol treatment. The completion rate of block A treatment was $70.8 \%$ (34/48). The breakdown of the 14 patients who discontinued treatment during block A was adverse events in 7 cases, PD in 4 cases, withdrawal of consent in 1 case, and other reasons in 2 cases. In addition, 1 patient deviated from protocol by continuing the treatment, and 1 patient discontinued due to hepatectomy after completing block A.

The rate of completion of block B treatment was $78.1 \%$ $(25 / 32)$. The breakdown of the seven patients who discontinued treatment during block B was PD in four cases, adverse event in one case, withdrawal of consent in one case, and hepatectomy in one case. A total of six patients with confirmed PD - three who discontinued block B and three who completed block B-were moved to block $\mathrm{C}$ as stipulated in the protocol. In addition, one patient discontinued due to withdrawal of consent after completing block B.

The rate of completion of block $\mathrm{C}$ treatment was $59.3 \%$ (16/27). The breakdown of the 11 patients who discontinued treatment during block $\mathrm{C}$ was $\mathrm{PD}$ in eight cases, adverse events in two cases, and withdrawal of consent in one case.

The protocol completion rate was $33.3 \%(16 / 48)$, and median TTF was 10.3 months (95\% confidence interval [CI], 5.6 to 12.1) (Fig. 4a).

The percentage of patients reintroduced to oxaliplatin at movement from block B to block C was $84.4 \%$ (27/32). 
Fig. 4 a Kaplan-Meier estimate of time to treatment failure. b Kaplan-Meier estimate of progression free survival a

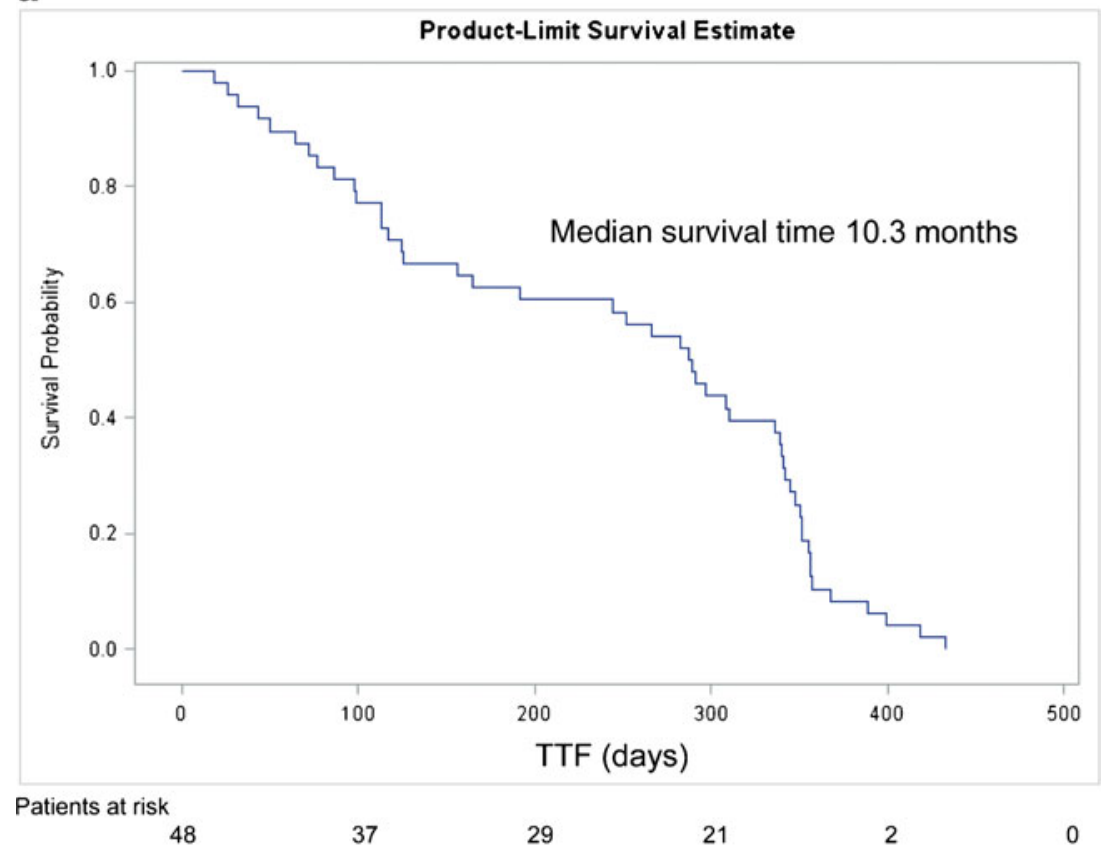

b

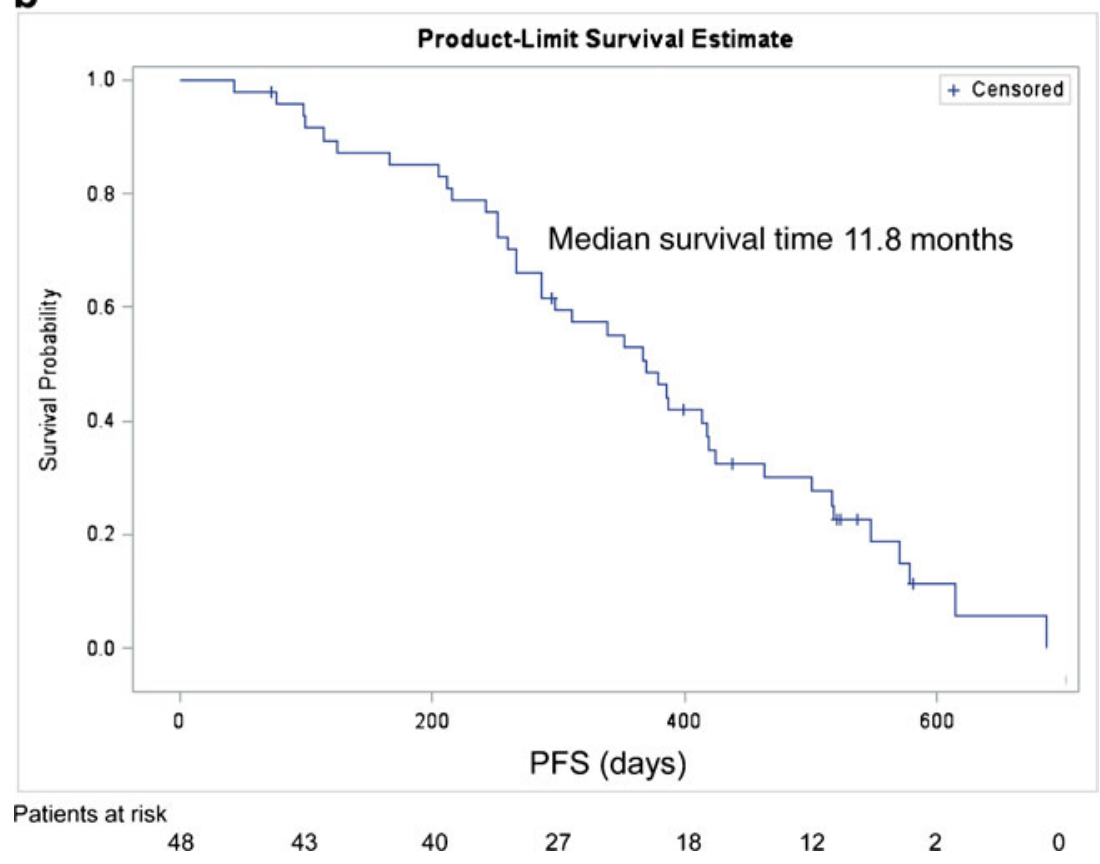

Relative dose intensity in each treatment block was $\geq 89 \%$ for 5 -FU and $l$-LV, $\geq 86 \%$ for bevacizumab, and $\geq 96 \%$ for oxaliplatin (blocks A and C).

\section{Efficacy}

The median PFS 1, was 11.8 months (95\% CI, 9.5 to 13.7) (Fig. 4b). The secondary endpoint RR 1 was $50 \%(23 / 46)$ in the 46 patients who were evaluable by RECIST. The maximum rate of tumor reduction at the time of best overall response during the protocol treatment was $-91 \%$ (range, +126.0 to $-91.0 \%$ ). There were six patients to whom PFS 2 and RR 2 were applicable after oxaliplatin reintroduction as patients with first PD during or immediately after block B treatment. The median PFS 2 and RR 2 were 4.2 months (95\% CI, 2.8 to 23.5$)$ and $0 \%(0 / 6)$, with three cases of stable disease (SD) and three cases of PD. Exploratory analysis showed median survival time to be 25.4 months (95\% CI, 18.5 to 35.2 ). 
Table 2 Frequency of common toxicities $(n=51$ : maximum toxicity per patient)

\begin{tabular}{lcccc}
\hline Adverse event & All grades $n(\%)$ & Grade 3 & Grade 4 & $\geq$ Grade $3(\%)$ \\
\hline Hematological & & & & $(2.0)$ \\
Leukopenia & $14(27.5)$ & 1 & 0 & $(7.8)$ \\
Neutropenia & $16(31.4)$ & 3 & 1 & $(0)$ \\
Thrombocytopenia & $13(25.5)$ & 0 & 0 & $(2.0)$ \\
Anemia & $45(88.2)$ & 0 & 1 & $(3.9)$ \\
Non-hematological & & & & $(2.0)$ \\
Peripheral neuropathy & $22(43.1)$ & 2 & 0 & $(3.9)$ \\
Allergic reaction & $5(9.8)$ & 1 & 0 & $(0)$ \\
Hypertension & $14(27.5)$ & 2 & 0 & $(0)$ \\
Proteinuria & $14(27.5)$ & 0 & - & $(3.9)$ \\
Hemorrhage & $1(2.0)$ & 0 & 0 & $(2.0)$ \\
Venous thromboembolism & $2(3.9)$ & 2 & 0 & $(0)$ \\
Nausea & $3(5.9)$ & 1 & 0 & $(2.0)$ \\
Vomiting & $4(7.8)$ & 0 & 0 & $(2.0)$ \\
Anorexia & $3(5.9)$ & 1 & 0 & $(2.0)$ \\
Ileus & $1(2.0)$ & 0 & 1 & $(2.0)$ \\
Diarrhea & $1(2.0)$ & 1 & 0 & 0 \\
Fatigue & $1(2.0)$ & 1 & 0 & \\
\hline
\end{tabular}

\section{Safety}

The main adverse drug reactions of grade 3 or higher in the 51 patients of the safety analysis set were neutropenia $7.8 \%$ (four patients), peripheral neuropathy $3.9 \%$ (two patients), hypertension $3.9 \%$ (two patients), venous thromboembolism $3.9 \%$ (two patients), and allergic reaction $2.0 \%$ (one patient). Grade 4 adverse drug reactions were neutropenia, decreased hemoglobin, and ileus, each $2.0 \%$ (one patient each). In addition, gastrointestinal perforation and clinically significant hemorrhage or proteinuria of grade 3 or higher, which are characteristic of bevacizumab, were not observed (Table 2). Total allergic reactions observed were $9.8 \%(5 / 51$ patients), which led to discontinuation of protocol treatment in three cases. Discontinuation of protocol due to peripheral neuropathy occurred in one case.

\section{Discussion}

This trial is the first investigational study of the oxaliplatin stop-and-go concept by a regimen of mFOLFOX7 (intermittent oxaliplatin administration) plus bevacizumab in Japanese patients with unresectable mCRC. This regimen was shown to be clinically useful for Japanese patients, with good tolerability and without impairing treatment efficacy.

Peripheral nerve disorders due to the cumulative toxicity of oxaliplatin are an obstacle to continuing FOLFOX. Therefore, a preplanned withdrawal of oxaliplatin for a period of 8 cycles was set in this study. In order to reduce hematological toxicity, mFOLFOX7 plus bevacizumab without 5-FU bolus administration was used, with bevacizumab co-administered with msLV5FU2 during the oxaliplatin withdrawal period.

Chemotherapy plus bevacizumab is recommended in the guidelines of various countries as the first-line therapy for unresectable mCRC. Comparing the therapeutic effects of different regimens in phase III studies with PFS as the primary endpoint, in the NO16966 study, PFS was 9.4 months in the FOLFOX4 plus bevacizumab group and was 9.3 months in the XELOX plus bevacizumab group [16], while in the HORIZON III study PFS in the mFOLFOX6 plus bevacizumab group was 10.3 months [17]. PFS in this study was 11.8 months, which is comparable to the results of those phase III studies. PFS in the intermittent administration group of the CONcePT trial [13] which preceded this study was 12.0 months, a similar result. Okita et al. [18] conducted mFOLFOX6 (intermittent oxaliplatin administration) plus bevacizumab by the stopand-go concept with Japanese patients as subjects, and the secondary endpoint PFS was 12.8 months, which is similar to the results of this study.

Preplanned withdrawal of oxaliplatin is one treatment strategy against peripheral neuropathy due to cumulative oxaliplatin toxicity. Peripheral nerve disorders of grade 3 or 4 occurred in $18 \%$ of the FOLFOX4 plus bevacizumab group in the NO16966 study [19] and in $10 \%$ of the mFOLFOX6 plus bevacizumab group in the HORIZON III study [17], but were substantially lower in this study at $3.9 \%$. Furthermore, peripheral neuropathy of grade 3 or 4 also occurred in $10 \%$ of patients in the intermittent administration groups in the CONcePT trial [13] and in the study 
reported by Okita et al. [18]. Therefore, the regimen of this study was very well tolerated by Japanese patients.

With respect to hematological toxicity, the incidence of neutropenia of grade 3 or 4 was $40 \%$ in the FOLFOX 4 plus bevacizumab group in the NO16966 study [19] and $24 \%$ in the mFOLFOX6 plus bevacizumab group in the HORIZON III study [17], but was lower in this study at $7.8 \%$. Okita et al. reported the incidence of neutropenia of grade 3 or 4 to be $40 \%$ [18], indicating that the mFOLFOX7 without bolus 5-FU regimen that we used in our study is good for keeping hematological toxicity low.

There are also some interesting study reports related to continuous oxaliplatin therapy. At the 2012 ASCO Annual Meeting, Yalcin et al. [20] compared a group that received continuous XELOX plus bevacizumab until PD with a group with preplanned withdrawal of oxaliplatin after six courses (18 weeks) of XELOX plus bevacizumab treatment and then continued on maintenance therapy of capecitabine plus bevacizumab. The primary endpoint PFS in the group that continued with capecitabine plus bevacizumab as maintenance therapy was significantly better than PFS in the group that continued with XELOX plus bevacizumab treatment until PD (capecitabine plus bevacizumab maintenance, 11.0 months [95\% CI, 9.1 to 12.9]; continuous XELOX plus bevacizumab, 8.3 months [95\% CI, 7.1 to 9.5$][P<$ $0.002])$. This result suggests the possibility that continued oxaliplatin treatment affects not only tolerability, but also maintenance of effectiveness.

Although the usefulness of the stop-and-go concept for unresectable mCRC is becoming established, including the results of this study, issues still remain from the standpoint of risk/benefit evaluation.

In representative studies of the stop-and-go concept, OPTIMOX1 [11] and OPTIMOX2 [12], oxaliplatin was stopped 12 weeks after the start of treatment, and Yalcin et al. [20] reported stopping oxaliplatin at 18 weeks after the start of treatment, while in this study, as in the CONcePT trial [13], oxaliplatin was stopped at 16 weeks after the start of treatment, and thus there are differences between studies. Because the cumulative maximum therapeutic effect reported by Haller [21] was after about 16 weeks ( 8 cycles) of FOLFOX4, and the incidence of peripheral neuropathy of grade 3 or higher due to oxaliplatin also increased, a preplanned withdrawal of oxaliplatin after about 16 weeks with the cumulative dose as guide seems to be most effective, but this has not yet been established. A clinical study [22] to resolve this as well as the timing for reintroducing oxaliplatin is currently underway, and we are awaiting the results.

Acknowledgments We thank the staff and patients of the following participating institutions: Chiba Medical Center, Chiba University, Chibaken Saiseikai Narashino Hospital, Fujita Health University
Hospital, Fukaya Red Cross Hospital, Kimitsu Chuo Hospital, Kumamoto University Hospital, Kurume University, Matsudo City Hospital, Minoh City Hospital, Nippon Medical School Chiba Hokusoh Hospital, Saishukan Hospital, Saitama Medical Center, Seirei Sakura Citizen Hospital, Teikyo University Chiba Medical Center, Toho University Sakura Medical Center, Tokyo Women's Medical University, Yokoyama Gastrointestinal Hospital, and ECRIN (Epidemiological and Clinical Research Information Network) for their support.

Conflict of interest We declare that we have no conflicts of interest.

Open Access This article is distributed under the terms of the Creative Commons Attribution License which permits any use, distribution, and reproduction in any medium, provided the original author(s) and the source are credited.

\section{References}

1. Petrelli N, Herreral L, Rustum Y, Burke P, Creaven P, Stulc J et al (1987) A prospective randomized trial of 5-fluorouracil versus 5fluorouracil and high-dose leucovorin versus 5-fluorouracil and methotrexate in previously untreated patients with advanced colorectal carcinoma. J Clin Oncol 5:1559-1565

2. de Gramont A, Bosset JF, Milan C, Rougier P, Bouche O, Etienne PL et al (1997) Randomized trial comparing monthly low-dose leucovorin and fluorouracil bolus with bimonthly high-dose leucovorin and fluorouracil bolus plus continuous infusion for advanced colorectal cancer: a French intergroup study. J Clin Oncol 15:808-815

3. de Gramont A, Figer A, Seymour M, Homerin M, Hmissi A, Cassidy J et al (2000) Leucovorin and fluorouracil with or without oxaliplatin as first-line treatment in advanced colorectal cancer. J Clin Oncol 18:2938-2947

4. Cheeseman SL, Joel SP, Chester JD, Wilson G, Dent JT, Richards FJ et al (2002) A 'modified de Gramont' regimen of fluorouracil, alone and with oxaliplatin, for advanced colorectal cancer. $\mathrm{Br} \mathrm{J}$ Cancer 87:393-399

5. Goldberg RM, Sargent DJ, Morton RF, Fuchs CS, Ramanathan RK, Williamson SK et al (2004) A randomized controlled trial of fluorouracil plus leucovorin, irinotecan, and oxaliplatin combinations in patients with previously untreated metastatic colorectal cancer. J Clin Oncol 22:23-30

6. Douillard JY, Cunningham D, Roth AD, Navarro M, James RD, Karasek P et al (2000) Irinotecan combined with fluorouracil compared with fluorouracil alone as first-line treatment for metastatic colorectal cancer: a multicentre randomised trial. Lancet 355:1041-1047

7. Hurwitz H, Fehrenbacher L, Novotny W, Cartwright T, Hainsworth J, Heim W et al (2004) Bevacizumab plus irinotecan, fluorouracil, and leucovorin for metastatic colorectal cancer. $\mathrm{N}$ Engl J Med 350:2335-2342

8. Kabbinavar FF, Hambleton J, Mass RD, Hurwitz HI, Bergsland E, Sarkar S (2005) Combined analysis of efficacy: the addition of bevacizumab to fluorouracil/leucovorin improves survival for patients with metastatic colorectal cancer. J Clin Oncol 23:3706-3712

9. Saltz LB, Clarke S, Diaz-Rubio E, Scheithauer W, Figer A, Wong $\mathrm{R}$ et al (2008) Bevacizumab in combination with oxaliplatin-based chemotherapy as first-line therapy in metastatic colorectal cancer: a randomized phase III study. J Clin Oncol 26:2013-2019

10. Goldberg RM, Sargent DJ, Morton RF, Fuchs CS, Ramanathan RK, Williamson SK et al (2006) Randomized controlled trial of reduced-dose bolus fluorouracil plus leucovorin and irinotecan or infused fluorouracil plus leucovorin and oxaliplatin in patients 
with previously untreated metastatic colorectal cancer: a North American Intergroup Trial. J Clin Oncol 24:3347-3353

11. Tournigand C, Cervantes A, Figer A, Lledo G, Flesch M, Buyse M et al (2006) OPTIMOX1: a randomized study of FOLFOX4 or FOLFOX7 with oxaliplatin in a stop-and-go fashion in advanced colorectal cancer-a GERCOR study. J Clin Oncol 24:394-400

12. Chibaudel B, Maindrault-Goebel F, Lledo G, Mineur L, Andre T, Bennamoun $\mathrm{M}$ et al (2009) Can chemotherapy be discontinued in unresectable metastatic colorectal cancer? The GERCOR OPTIMOX2 Study. J Clin Oncol 27:5727-5733

13. Grothey A, Hart LL, Rowland KM, Ansari RH, Alberts SR, Chowhan NM et al (2008) Intermittent oxaliplatin (oxali) administration and time-to-treatment failure (TTF) in metastatic colorectal cancer (mCRC): Final results of the phase III CONcePT trial. J Clin Oncol 26(suppl 15S):180s, abstr 4010

14. Hecht JR, Mitchell E, Chidiac T, Scroggin C, Hagenstad C, Spigel D et al (2008) An updated analysis of safety and efficacy of oxaliplatin (Ox)/bevacizumab (bev) $+/$ - panitumumab (pmab) for first-line treatment (tx) of metastatic colorectal cancer (mCRC) from a randomized, controlled trial (PACCE). Presented at the Am Soc Clin Oncol GI Cancer Symposium (abstr 273)

15. Colucci G, Gebbia V, Paoletti G, Giuliani F, Caruso M, Gebbia N et al (2005) Phase III randomized trial of FOLFIRI versus FOLFOX4 in the treatment of advanced colorectal cancer: a multicenter study of the Gruppo Oncologico Dell'Italia Meridionale. J Clin Oncol 23:4866-4875

16. Cassidy J, Clarke S, Diaz-Rubio E, Scheithauer W, Figer A, Wong $\mathrm{R}$ et al (2008) Randomized phase III study of capecitabine plus oxaliplatin compared with fluorouracil/folinic acid plus oxaliplatin as first-line therapy for metastatic colorectal cancer. J Clin Oncol 26:2006-2012

17. Schmoll HJ, Cunningham D, Sobrero A, Karapetis CS, Rougier P, Koski SL et al (2012) Cediranib with mFOLFOX6 versus bevacizumab with mFOLFOX6 as first-line treatment for patients with advanced colorectal cancer: a double-blind, randomized phase III study (HORIZON III). J Clin Oncol 30:3588-3595

18. Okita NT, Esaki T, Baba E, Sakai D, Tokunaga S, Takiuchi H et al (2012) A multicenter phase II study of the stop-and-go modified FOLFOX6 with bevacizumab for first-line treatment of patients with metastatic colorectal cancer. Invest New Drugs 30:2026-2031

19. Cassidy J, Clarke S, Diaz-Rubio E, Scheithauer W, Figer A, Wong $\mathrm{R}$ et al (2011) XELOX vs FOLFOX-4 as first-line therapy for metastatic colorectal cancer: NO16966 updated results. Br J Cancer 105:58-64

20. Yalcin S, Uslu R, Dane F, Yilmaz U, Zengin N, Buyukunal E et al (2012) Bevacizumab (BEV) plus capecitabine as maintenance therapy after initial treatment with BEV plus XELOX in previously untreated patients (pts) with metastatic colorectal cancer (mCRC): Mature data from STOP and GO, a phase III, randomized, multicenter study. J Clin Oncol 30 (suppl 15s): abstr 3565

21. Haller DG (2000) Safety of oxaliplatin in the treatment of colorectal cancer. Oncology (Williston Park) 14(12 suppl 11):15-20

22. Maintenance treatment versus observation after induction in advanced colorectal carcinoma (CAIRO3). [Cited 29/03/2013.] Available from URL: http://clinicaltrials.gov/ct2/show/NCT00442637 